\title{
Effects of Porphyra yezoensis Polysaccharide with Different Molecular Weights on the Adhesion and Endocytosis of Nanocalcium Oxalate Monohydrate in Repairing Damaged HK-2 Cells
}

\author{
Hui Zhang, Xin-Yuan Sun*, Jian-Ming Ouyang* \\ Institute of Biomineralization and Lithiasis Research, Jinan University, No.601, Huangpu \\ Avenue West, Tianhe District, Guangzhou, China. 510632
}

*Corresponding authors. E-mail address: sunxinyuan1985@163.com (Xin-Yuan Sun) and toyjm@jnu.edu.cn (Jian-Ming Ouyang)

Number of pages: 3

Number of figures: 1

Number of tables: 1 
Table S1. Degradation conditions and chemical properties of original and degraded P. yezoensis polysaccharides

\begin{tabular}{cccc}
\hline & Molecular weight $\mathrm{M}_{\mathrm{w}} /$ & $-\mathrm{OSO}_{3} \mathrm{H}$ content & -COOH content \\
Sample abbreviation & $\mathrm{kDa}$ & $/ \%$ & $/ \%$ \\
\hline PYP0 & $4669 \pm 214$ & 17.30 & 1.25 \\
PYP1 & $576.2 \pm 13.3$ & 17.51 & 1.42 \\
PYP2 & $49.54 \pm 2.70$ & 17.69 & 1.53 \\
PYP3 & $12.65 \pm 1.04$ & 17.83 & 1.66 \\
PYP4 & $4.02 \pm 0.58$ & 17.86 & 1.70 \\
\hline
\end{tabular}




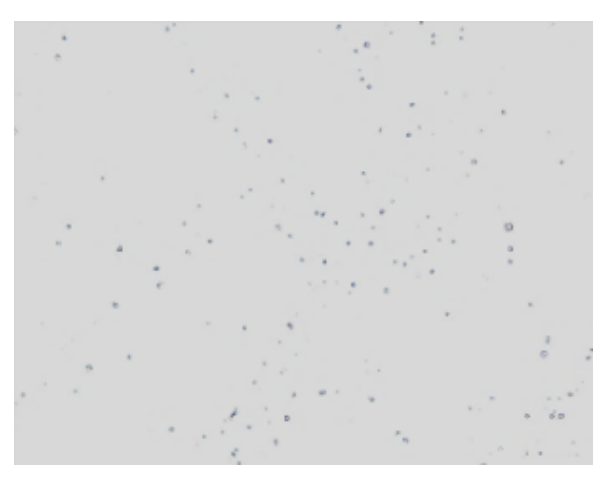

(A)

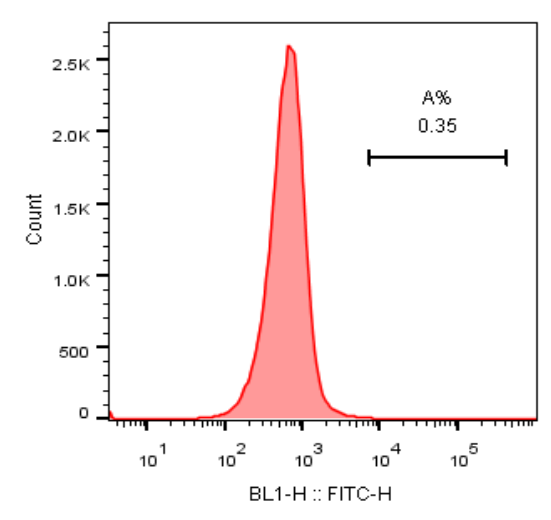

(C)

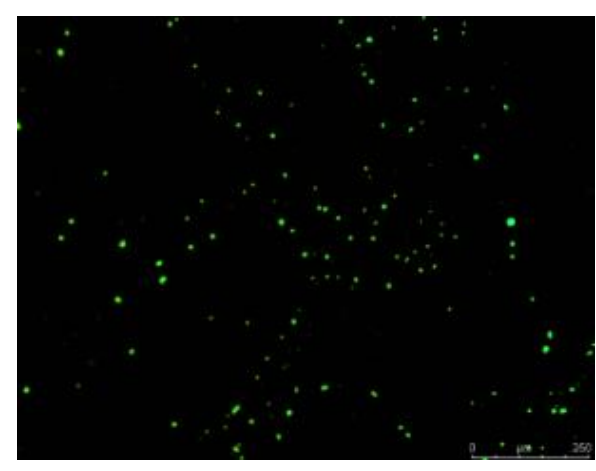

(B)

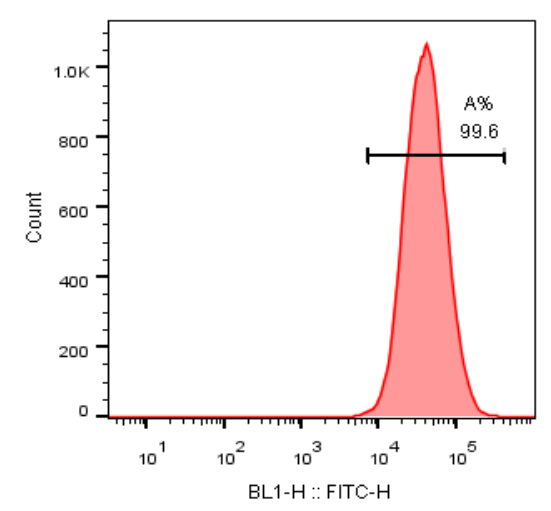

(D)

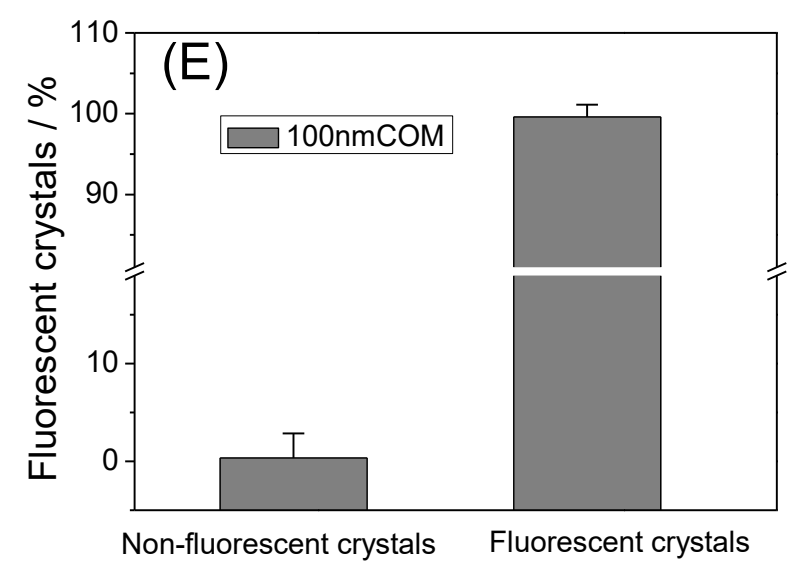

Figure S1. FITC fluorescent labeling of nano-COM crystals. Optical microscope images of COM crystal before and after fluorescent labeling (A, B); Flow cytometric analysis histogram of fluorescent ratio of plain and FITC fluorescent labeled nano COM crystal (C, D); Statistics histogram of the percentage of fluorescent labeled COM crystals (E). 